\section{A beige immune response}

defects in
beige fat
biogenesis
were reported
by both
groups in mice
that lacked
signalling
through
the shared
subunit of the
receptors for
IL-4 and IL-13

Brown adipose tissue (BAT) is specialized for the dissipation of chemical energy in the form of heat, a process that is dependent on mitochondrial uncoupling protein 1 (UCP1). Adult humans lack classical BAT but they have a subset of cold-inducible 'beige' adipocytes in their white adipose tissue (WAT). There is currently great interest in defining the pathways by which beige fat is induced, as this could have therapeutic applications for obesity and related metabolic disorders. Two papers published in Cell describe how type 2 immune responses can promote beige fat thermogenesis in response to both cold and exercise.

Rao et al. identified meteorin-like protein (METRNL) as a secreted factor that is induced in skeletal muscle by resistance exercise in mice and humans. Of note, METRNL expression was also induced in adipose tissue by cold exposure. Increased expression of METRNL systemically or in adipose tissue resulted in 'browning' of the WAT, as shown by the increased expression of thermogenic genes such as Ucp 1. However, METRNL had no direct effect on the thermogenesis of white adipocytes

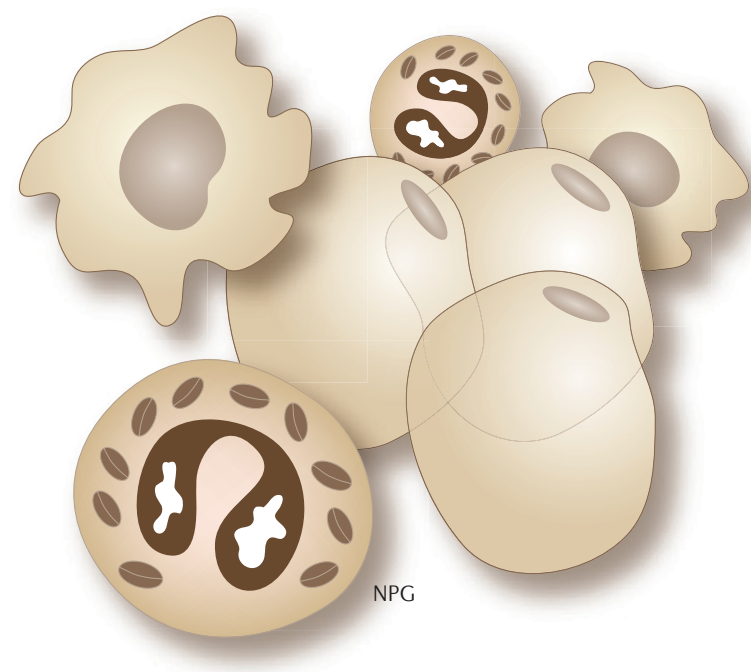

in vitro, which indicated the involvement of a non-adipose cell type in the induction of beige fat.

In support of such an indirect effect - and building on previous work by Nguyen, Qiu et al. which showed that cold exposure induces alternative macrophage activation in an interleukin-4 (IL-4)- and IL-13-dependent manner - both studies identified a role for alternatively activated macrophages in the 'browning' pathway. METRNLtreated mice (Rao et al.) and mice exposed to prolonged cold (Qiu et al.) had increased macrophage numbers in WAT and increased expression of genes that are associated with alternative macrophage activation, such as the gene encoding arginase 1 . Qiu et al. further showed that the increased number of macrophages and the expression of thermogenic proteins, such as UCP1, in adipose tissue after cold exposure depend on the CC-chemokine receptor 2 (CCR2)-dependent recruitment of blood monocytes.

Qiu et al. showed that signalling by the type 2 cytokines IL- 4 and IL-13 is required for cold-induced beige fat thermogenesis; $I l 4^{-/-} I l 13^{-/-}$mice had decreased expression of $U c p 1$ and decreased thermogenic capacity after cold exposure. Defects in beige fat biogenesis were reported by both groups in mice that lacked signalling through the shared subunit of the receptors for IL-4 and IL-13 (IL-4Ra) or its downstream transcription factor signal transducer and activator of transcription 6 (STAT6). Both groups identified eosinophils as being responsible for the type 2 cytokine production that was required for the 'browning' pathway. Rao et al. showed that systemic administration of METRNL increases the number of adipose tissue eosinophils, which are required for the induction of Ucp1, and Qiu et al. showed that coldinduced UCP1 protein expression in WAT was attenuated in mice lacking eosinophils.

Together, the studies show that cold exposure and resistance exercise induce a canonical type 2 immune response involving IL-4, IL-13 and eosinophils that leads to the alternative activation of adipose tissue macrophages. Alternatively activated macrophages can induce $\mathrm{UCP} 1^{+}$beige adipocytes through catecholamine secretion. Cold exposure or METRNL expression significantly increased macrophage expression of tyrosine hydroxylase (the rate-limiting enzyme for catecholamine synthesis) in an IL-4-, IL-13-, IL-4R $\alpha$ - and STAT6-dependent manner.

The therapeutic potential of this pathway was illustrated by Qiu et al., who showed that treating thermoneutral mice with IL- 4 increased the expression of arginase 1, catecholamines and UCP1 in the WAT, and increased their basal metabolic rate. Mice that were fed a high-fat diet had decreased expression of tyrosine hydroxylase and UCP1 in the WAT, which could be restored by IL- 4 therapy, leading to weight loss and improved insulin sensitivity. Thus, the role of type 2 cytokines in inducing the beige fat thermogenic pathway links the regulation of immune responses to energy homeostasis.

Kirsty Minton

ORIGINAL RESEARCH PAPERS Rao, R. R. et al. Meteorin-like is a hormone that regulates immune-adipose interactions to increase beige fat thermogenesis. Cell http://dx.doi. org/10.1016/j.cell.2014.03.065 (2014) | Qiu, Y. et al. Eosinophils and type 2 cytokine signaling in macrophages orchestrate development of functional beige fat. Cell $\underline{h t t p: / / d x . d o i . ~}$ org/10.1016/j.cell.2014.03.066 (2014) 